\title{
Une promenade extraterrestre avec Marc Boulay
}

\section{A Stroll Through Outer Space with Marc Boulay}

\author{
Marc Boulay ${ }^{1}$ \\ ${ }^{1}$ artiste indépendant : Auteur, concept artist, 3D creature designer
}

RÉSUMÉ. L'artiste Marc Boulay nous invite ici à une promenade extraterrestre graphique entre ses thèmes de prédilection et de rêverie d'exobiologie spéculative. II s'agit d'un ensemble de " photos » et de croquis rapportés de ses voyages imaginaires et extraits de sa nouvelle production: "Carnets de Voyages Stellaires - Souvenirs de Terrains ». II a associé à ses " photos » et ses croquis des commentaires particulièrement originaux.

ABSTRACT. The artist Marc Boulay has invited us to embark on a visual interstellar promenade, strolling amongst his favourite subjects and dreams of speculative exobiology. Here is a collection of "snapshots" and sketches from the voyages of the imagination featured in his latest book, Carnets de Voyages Stellaires - Souvenirs de Terrains. Highly original text forms the commentary for the snapshots and sketches.

MOTS-CLÉS. animaux imaginaires, art, extraterrestres.

KEYWORDS. fictitious animals, art, aliens.

Marc Boulay est auteur, concept artist, 3D creature designer et voyageur immobile. Après de nombreux allers-retours dans le Temps, de voyages dans le passé avec ses reconstitutions paléontologiques et d'explorations dans le futur avec « Krakken, Future Ocean » et « Demain, Les Animaux du Futur », il ne lui restait plus que l’Espace comme terrain de jeu. Créatif acharné, Marc imagine et développe constamment de nouveaux mondes, de nouvelles planètes et d'étranges écosystèmes à la fois improbables et cohérents. De son home studio, il travaille depuis 2016 sur un thème qu'il affectionne particulièrement : l'exobiologie spéculative. Il imagine, modélise et réalise alors : «Another World», «Another World2», «Carnet de Voyages Stellaires - Souvenirs de Terrains » et autres «Cartes Postales Interstellaires »... où il propose des créations originales, images et vidéos photo-réalistes $(4 \mathrm{k}, 8 \mathrm{k} \& 12 \mathrm{k}) \mathrm{d}$ 'organismes et d'écosystèmes extraterrestres tout droit sortis de son imaginaire. Imaginaire enrichi de données scientifiques, bien sûr. Marc a participé à de nombreux ouvrages, expositions, conférences, concept-arts innovants, réalisations muséographiques et cinématographiques grands formats (I-Max, 360-8k/12k) ayant comme thème principal l'évolution de la biodiversité passée et future terrestre... et celles d'autres mondes.

Parfois, il sort de sa bulle spatio-temporelle pour participer à quelques missions scientifiques et artistiques internationales (Maroc, Laos, Chine...) au cours desquelles il affine son art et ses connaissances dans ses domaines de prédilection que sont les arts, les sciences et la diffusion des connaissances. Ainsi, en 2020/2021, après 10 mois d'isolement total, il a réalisé, en $360^{\circ} / 12 \mathrm{k}$, la scène finale de «Cartes postales interstellaires » du Planétarium de La Cité Des Sciences et de l'Industrie de Paris. En 2017/2018, Marc produit lui-même son film expérimental sur l'exobiologie spéculative "Another World». A sa grande surprise et sans attentes particulières, son film est sélectionné, section court métrage Art \& Science, par le jury du Festival International du Film Scientifique de Paris, Pariscience. En 2017, il s'est immergé 75 jours en Chine à Longzhou, où il était invité à une résidence d'artistes. Durant son séjour, il a présenté trois de ses œuvres sur le thème de l'exobiologie spéculative à l'Exposition des Artistes Français au Musée d'Art contemporain de Nanning/Chine. En 2017, il a été invité au Festival International de la Photographie Animalière de Montier-En-Der où il a exposé, à la Halle au Blé, 30 de ses œuvres sur l'exobiologie spéculative et projeté, pour la première fois au public, son court métrage «Another World». En 2016, il a co-signé le livre « Combien de doigts a un extraterrestre ?» avec l'astrophysicien Roland Lehoucq et le paléontologue Jean-Sébastien Steyer (Lehoucq/Steyer/Boulay, Eds Belin). Entre 2004 à 2015, il entreprend son voyage dans le futur. En 2015, il revient furtivement de son voyage et 
publie «Demain, Les Animaux du Futur» qu'il co-signe avec avec Jean-Sébastien Steyer (Boulay/Steyer, Editions Belin).

Et avant ? Il était sculpteur animalier et paléoartiste. Ses images sont encore aujourd'hui dans de nombreux livres, couvertures de livres et autres covers musicaux et ses sculptures intemporelles sont toujours les pièces maîtresses des plus grands musées et institutions d'Europe : Grande Galerie de l'Évolution, Palais de la découverte, Cité des Sciences et de l'Industrie de Paris, Institut royal des Sciences naturelles de Bruxelles.

\section{Bienvenue sur Moon A02!}

Moon A02 est l'une des 3 lunes gravitant autour d'une géante gazeuse qui gravite elle-même autour de sa naine rouge.

C'est la plus petite et la plus peuplée des 3 lunes. Son océan d'eau sulfurée lui confère une atmosphère particulièrement saturée de sulfure d'hydrogène rendant toutes captures d'images impossibles, difficiles ou altérées.

Autour des naines rouges peu lumineuses, la "végétation", adaptée pour absorber toutes les longueurs d'ondes de la lumière visible, pourrait paraître presque noire, à nos yeux.

Voici quelques photos en monochrome sépia que j'ai réussi à sauver des réactions chimiques atmosphériques.

Note : Alors que j'étais en voyages stellaires, le Planétarium de La Cité des Sciences de Paris, s'est réservé quelques séquences filmées de Moon A02.

La totalité du bestiaire (faune /flore) et autres paysages de cette petite lune ne figure pas, bien sûr, sur ces quelques minutes de film.

J'ai répertorié environ 500 espèces soigneusement conservées et protégées des regards indiscrets sur 4 térabits de disques durs. 


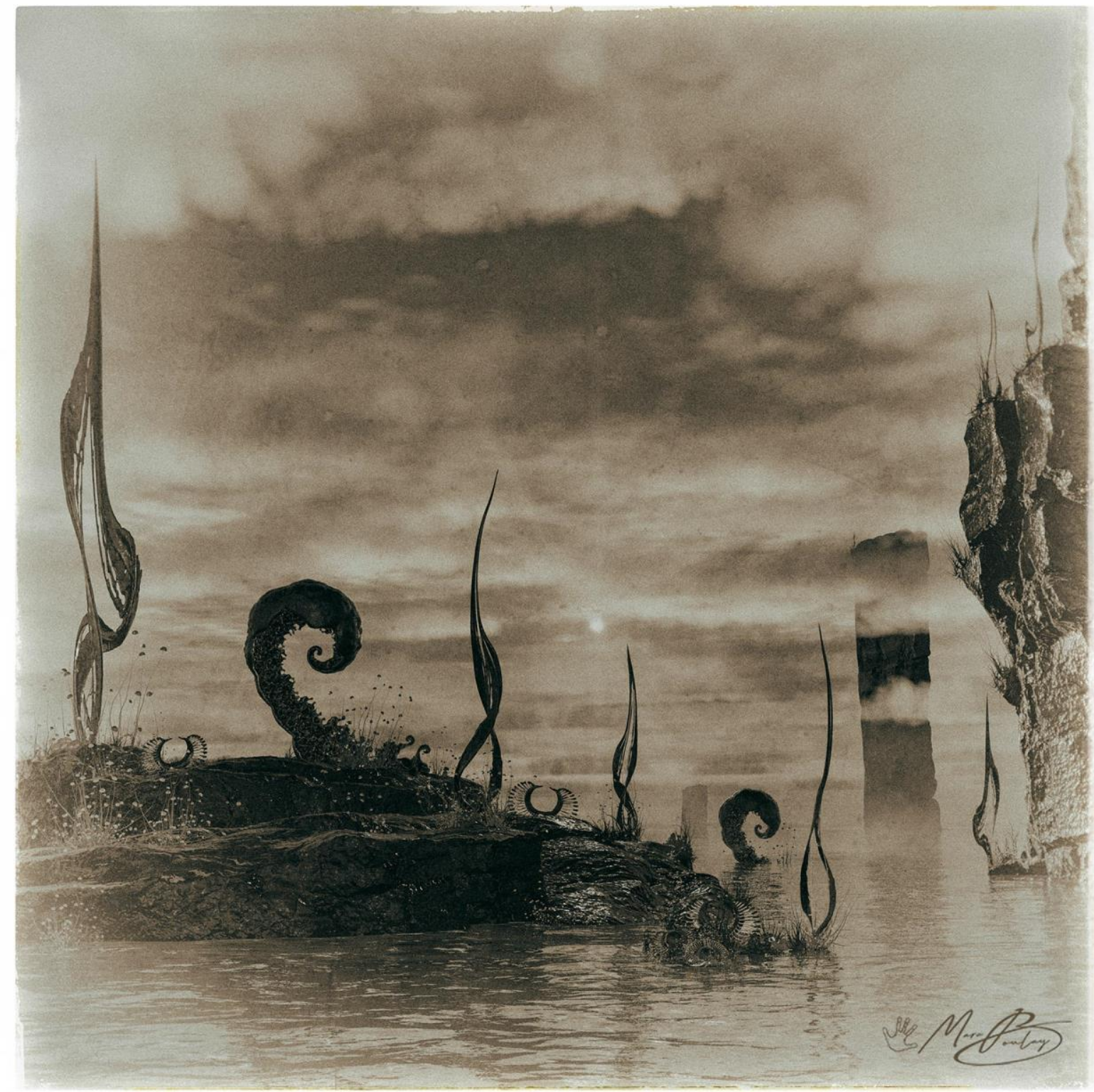

\section{2. "Carnets de Voyages Stellaires - Souvenirs de Terrains" Sp.22}

Sp : Abréviation de species au singulier utilisée pour indiquer que l'espèce (animal ou plante), dont on donne le nom de genre n'a pas été identifiée avec plus de précision et "22" parce que c'est la 22ème espèce du "genre" à avoir été découverte sur Moon A02.

Quel que soit l'endroit où se pose le regard, il est difficile de ne pas remarquer cette espèce.

Sp.22 est présente aussi bien sur les rivages de l'océan sulfuré que posé à différents niveaux de l'océan. Certains individus ont même été observés à plus de $1000 \mathrm{~m}$ de profondeur !

Cette espèce à la fois organique et minérale présente un exosquelette de silicium et d'architecture très complexe au centre duquel se loge une sorte de noyau recouvert d'une membrane souple et très épaisse. On peut distinguer au travers de cette membrane un système organique parfaitement réactif à son environnement. La plupart des spécimens ne dépassent pas $50 \mathrm{~cm}$.

Cette espèce, comme la plupart des espèces de Moon A02, émet un flux de lumière bioluminescente dès qu'elle se sent menacée et pour, vraisemblablement, communiquer entre individus du groupe. Il semblerait même que Sp.22 puisse communiquer avec d'autres espèces du même biome. 
Les plus jeunes ou du moins les plus petites (- de 10cm) ont été observées en pleine eau, dérivant au grès des courants marins jusqu'à ce qu'elles puissent se fixer à une surface rocheuse.

Note : L'architecture à la fois "simple" et très complexe de Sp.22, a suscité de vif l'intérêt de la part de certains joailliers.

Il est vrai qu'un bijou inspiré d'une forme de vie extraterrestre n'est pas très courant sur la place Vendôme!

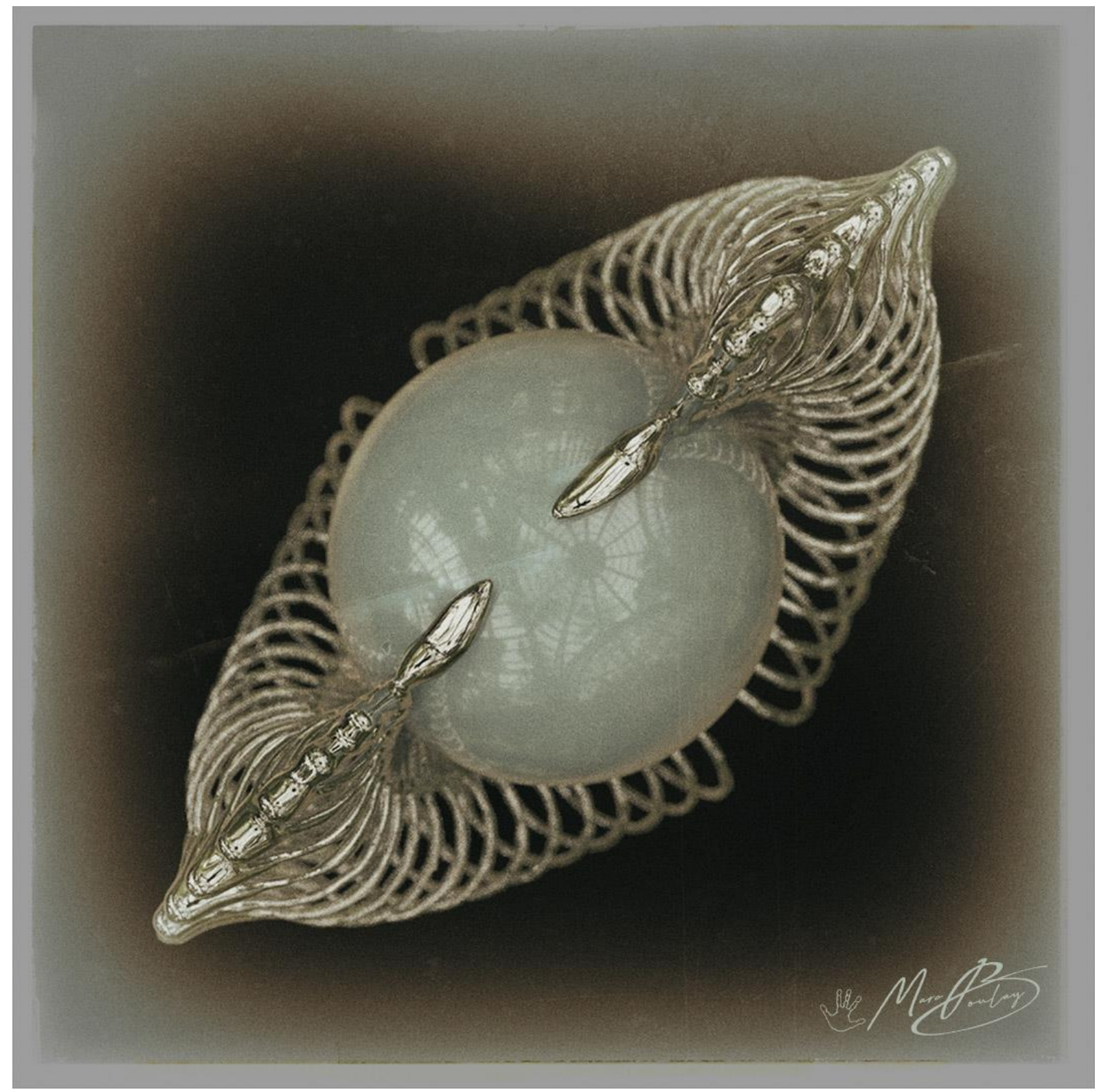



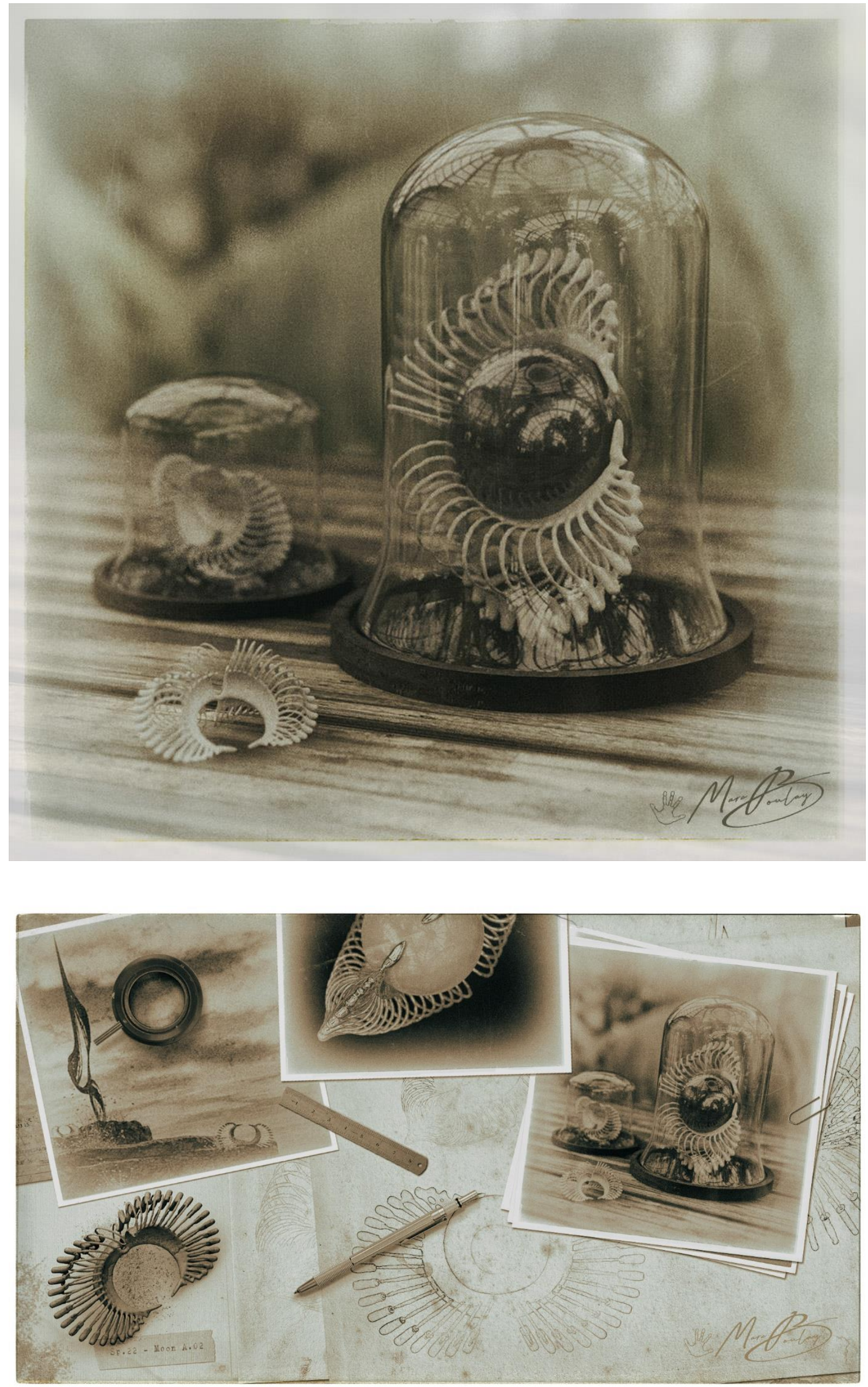


\section{3. "Carnets de Voyages Stellaires - Souvenirs de Terrains" Sp.12}

Sp.12 est très certainement l'espèce emblématique de Moon A02

Sur la globalité de cette lune, les paysages ont tous cette signature graphique particulière dessinée par quelques groupes ou spécimens isolés de cette bien étrange espèce nommée Sp.12

Cet organisme pousse là où ses crampons peuvent la fixer. Cette espèce à la fois minérale, végétale et animale s'élève à plusieurs mètres voire dizaines de mètres de hauteur.

L'endosquelette - non encore étudié - est visible sur les plus jeunes spécimens.

Sp.12 "pousse" généralement en plaine par petits groupes de 2 ou 3, cependant, j'ai pu observer quelques individus solitaires sur certains reliefs tabulaires résiduels à des altitudes comprises entre 500 à $2000 \mathrm{~m}$.
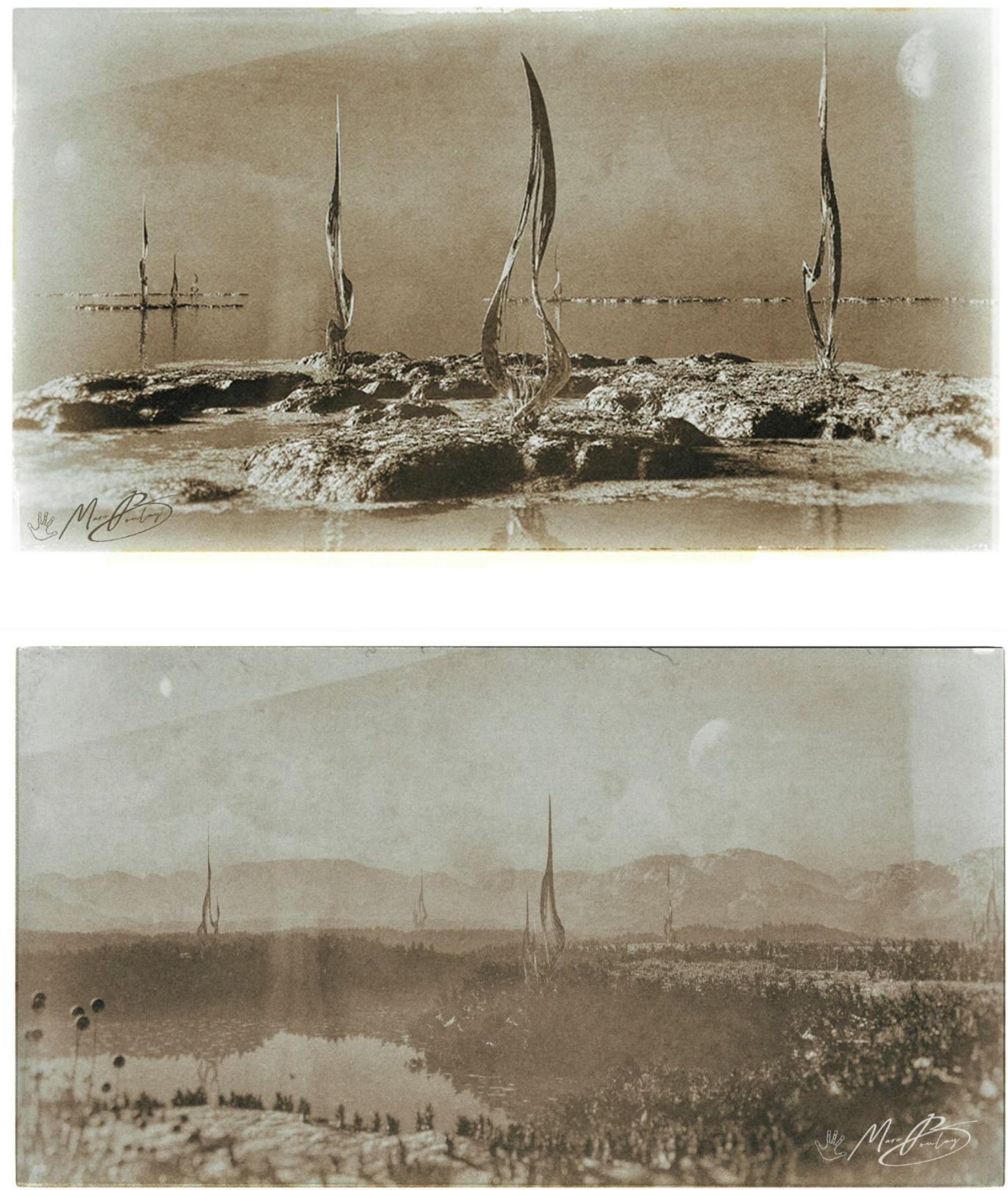


\section{4. "Carnets de Voyages Stellaires - Souvenirs de Terrains" Sp.10}

J'ai rencontré cette petite espèce aquatique dans les eaux sulfureuses de quelques lacs naturels.

Dans son milieu, cet organisme pélagique de quelques dizaines de centimètres émet des flashs bioluminescents de couleurs variées. Il semblerait que les gammes de couleurs correspondent au niveau de profondeur où il se trouve. La fréquence et l'intensité des flashs pourraient être une forme de communication intraspécifique de cette espèce. Des observations approfondies in situ permettraient de confirmer cette hypothèse.

D'apparence gélatineuse, Sp.10 est dépourvus de squelette, de cerveau et de poumon mais, en réalité, leur structure est très complexe.

Sp.10 possèdent des structures sensorielles très élaborées comme des ocelles rassemblées en périphérie de leur rhopalie (rhopalie est une zone de concentration d'organes récepteurs présente chez les scyphozoaires et les cubozoaires terrestres)

A ma grande surprise, cet organisme se solidifié à l'air libre. Probablement une réaction de défense en milieu hostile cumulé à une réaction chimique entre les composants de son derme et les sulfures d'hydrogène présents dans l'atmosphère. Quoi qu'il en soit, Sp.10 ne meurt pas pour autant et semblerait se mettre dans une sorte de "diapause" jusqu'à ce que son entourage lui semble à nouveau propice.

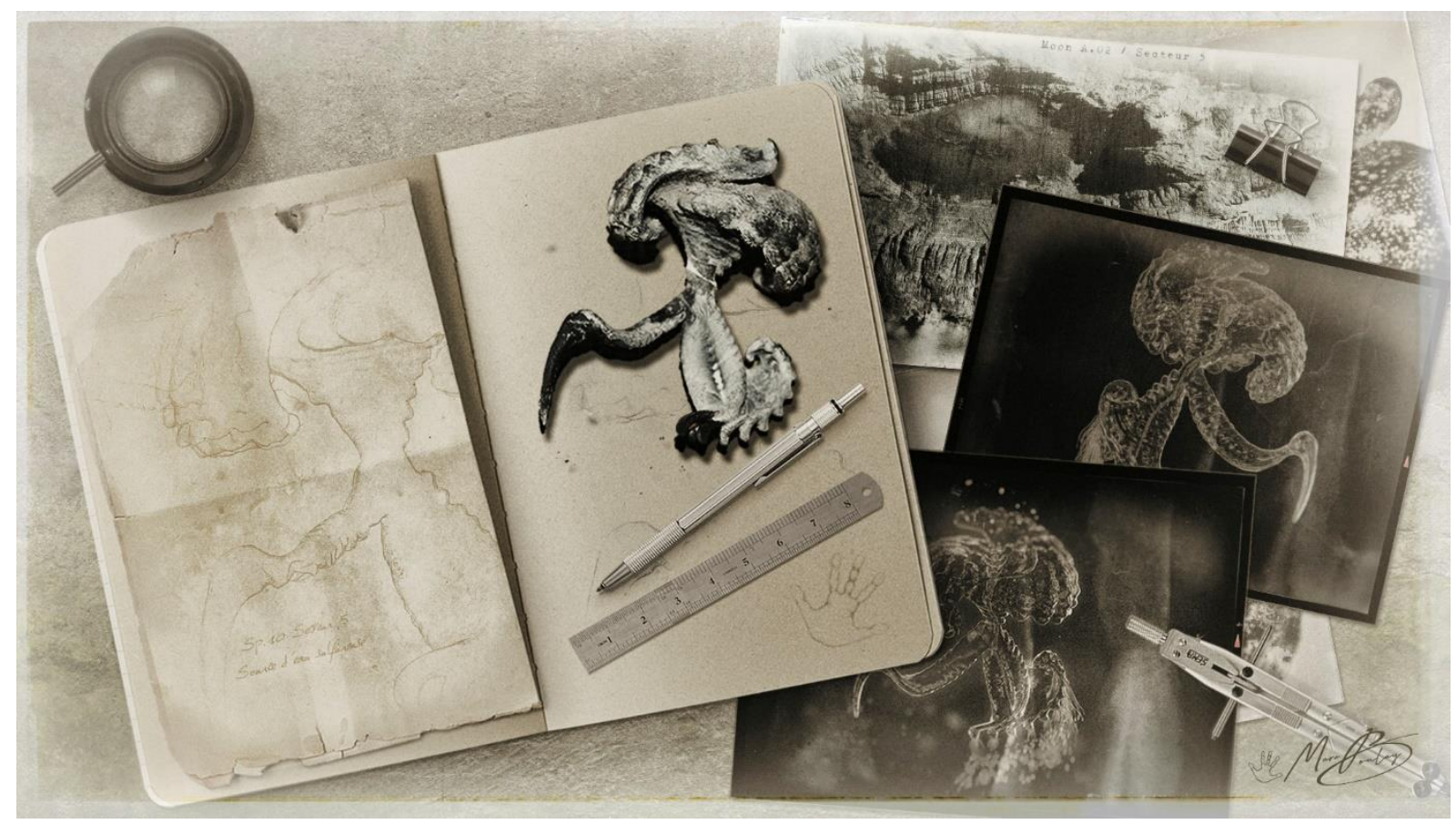




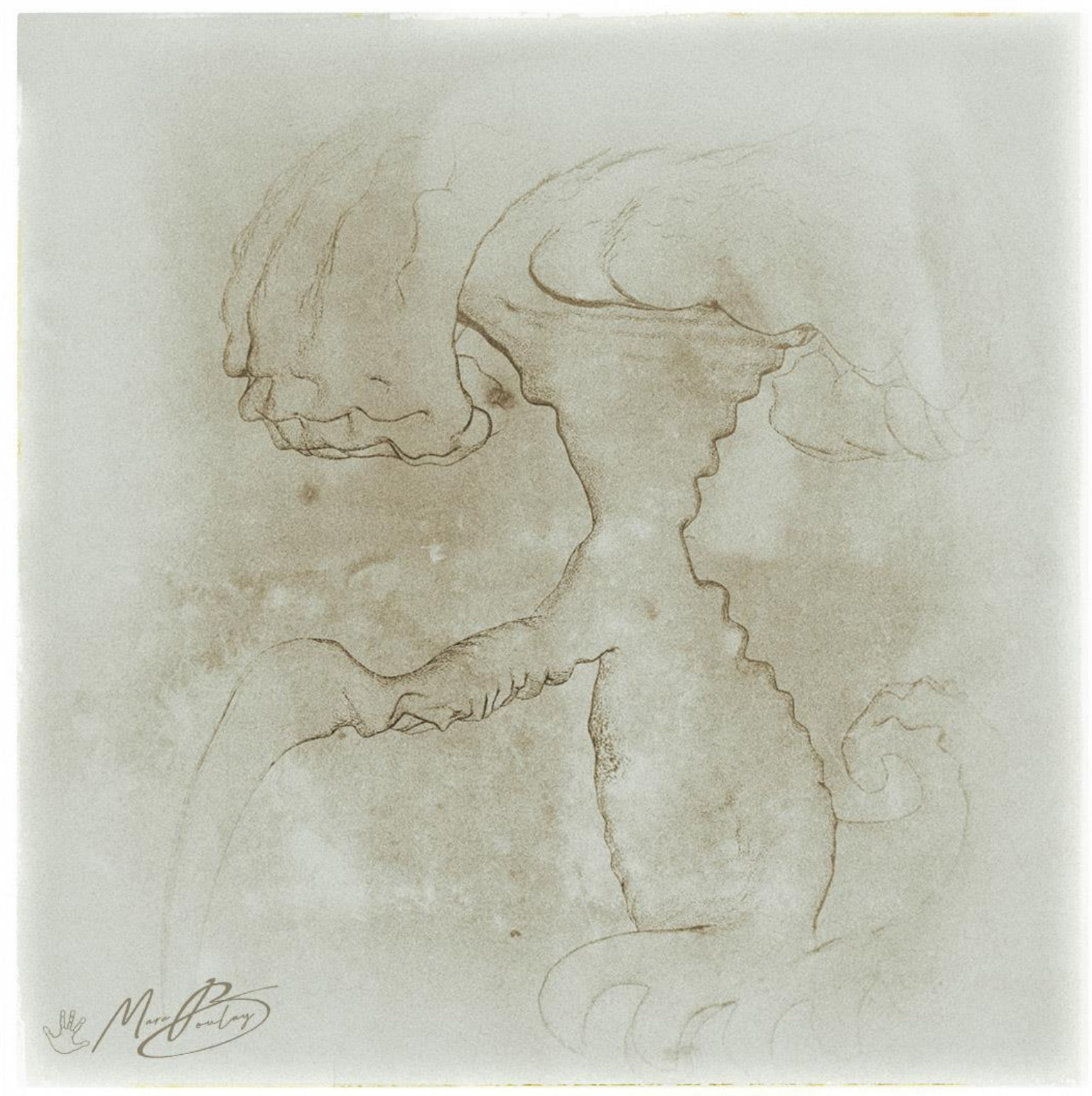




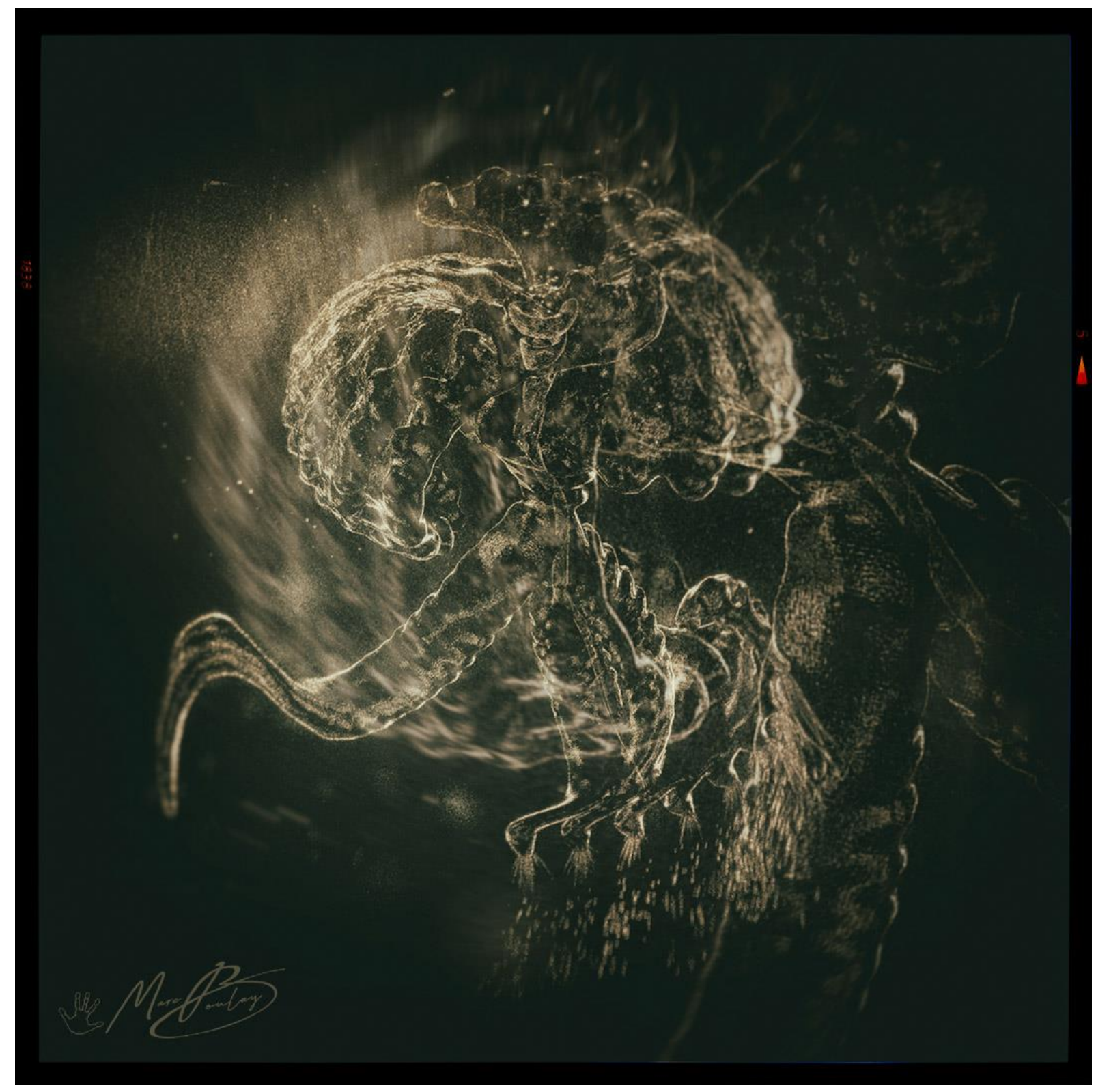

\section{5. "Carnets de Voyages Stellaires - Souvenirs de Terrains" Sp.700}

Voici 2 clichés de Sp.700 réalisés dans les eaux profondes et cristallines d'un petit lac sulfuré de Moon A02.

A première vue, cette espèce présente les caractéristiques des cnidaires terrestres. Cependant, celles-ci sont bien plus grandes et semblent être l'espèce dominante dans ce petit lac.

En raison de leurs nématocystes (filaments capables de lancer des harpons venimeux), je n'ai pas pu m'approcher pour les observer.

Sur Moon A02 comme sur Terre, il semblerait qu'il y ait au moins 2 formes dans ce "groupe". L'une fixée au substrat et l'autre, comme celle-ci, libre et mobile. 


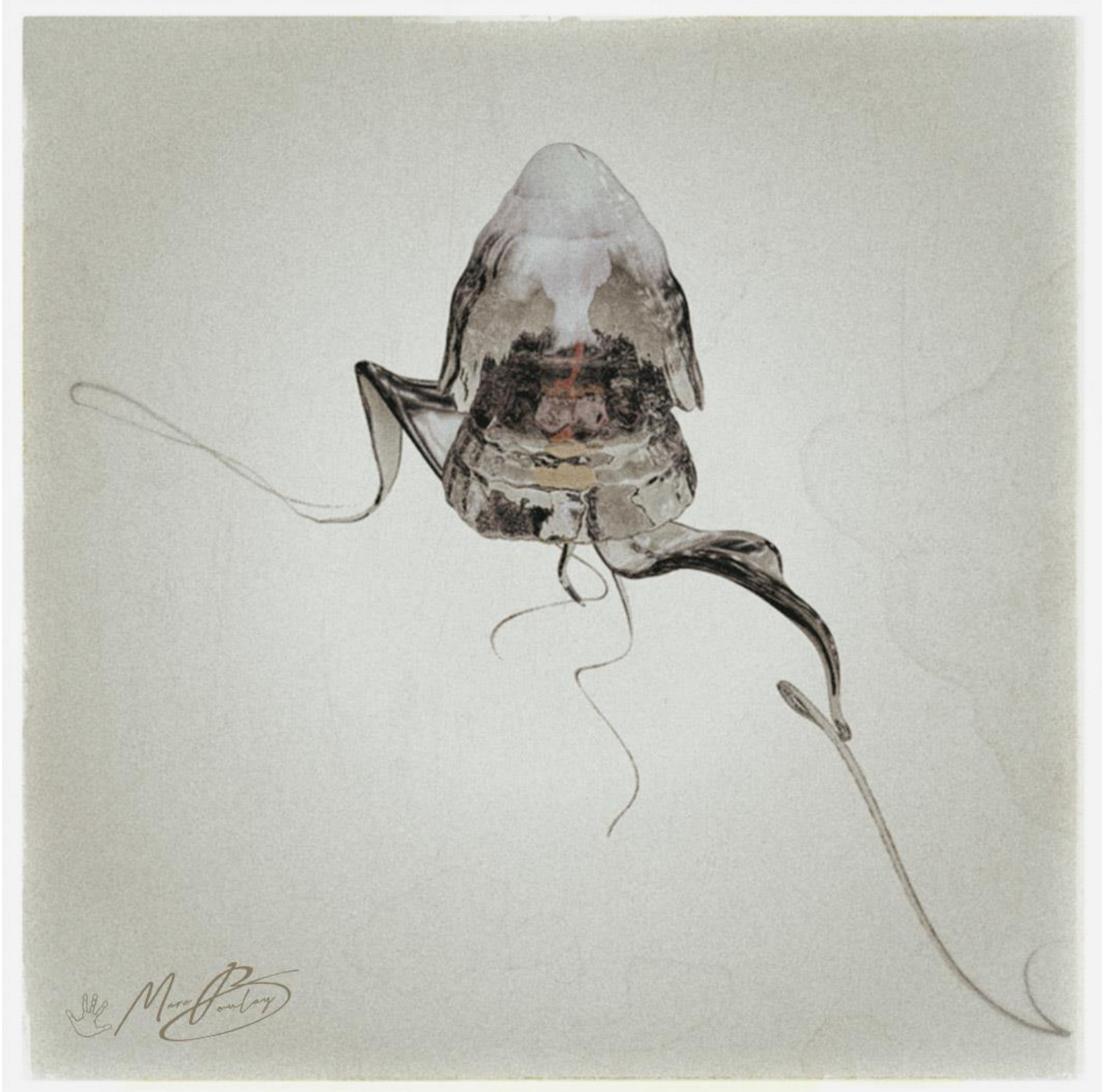




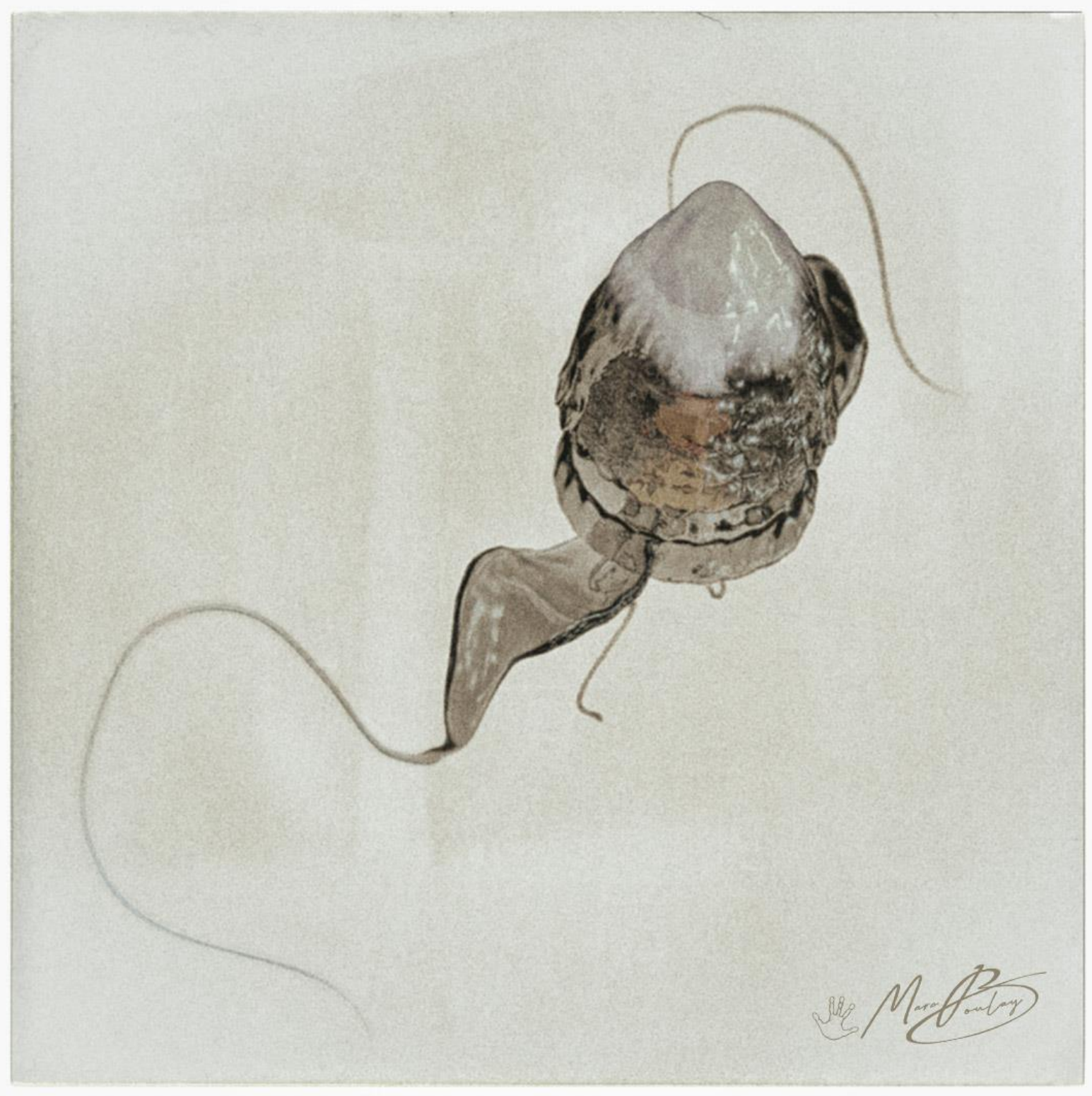

6. "Carnets de Voyages Stellaires - Souvenirs de Terrains" Sp.704

Sur ces 2 clichés et une esquisse - sauvés des eaux acides de l'océan sulfuré - voici Sp.704 qui ne ressemble à rien de connu sur Terre.

J'ai tenté de dessiner son architecture très particulière afin de représenter ses divers emboîtements qui pourraient être des anneaux de croissances.

Sp.704 est une petite espèce pélagique (de pleine mer) qui vit près de la surface. 


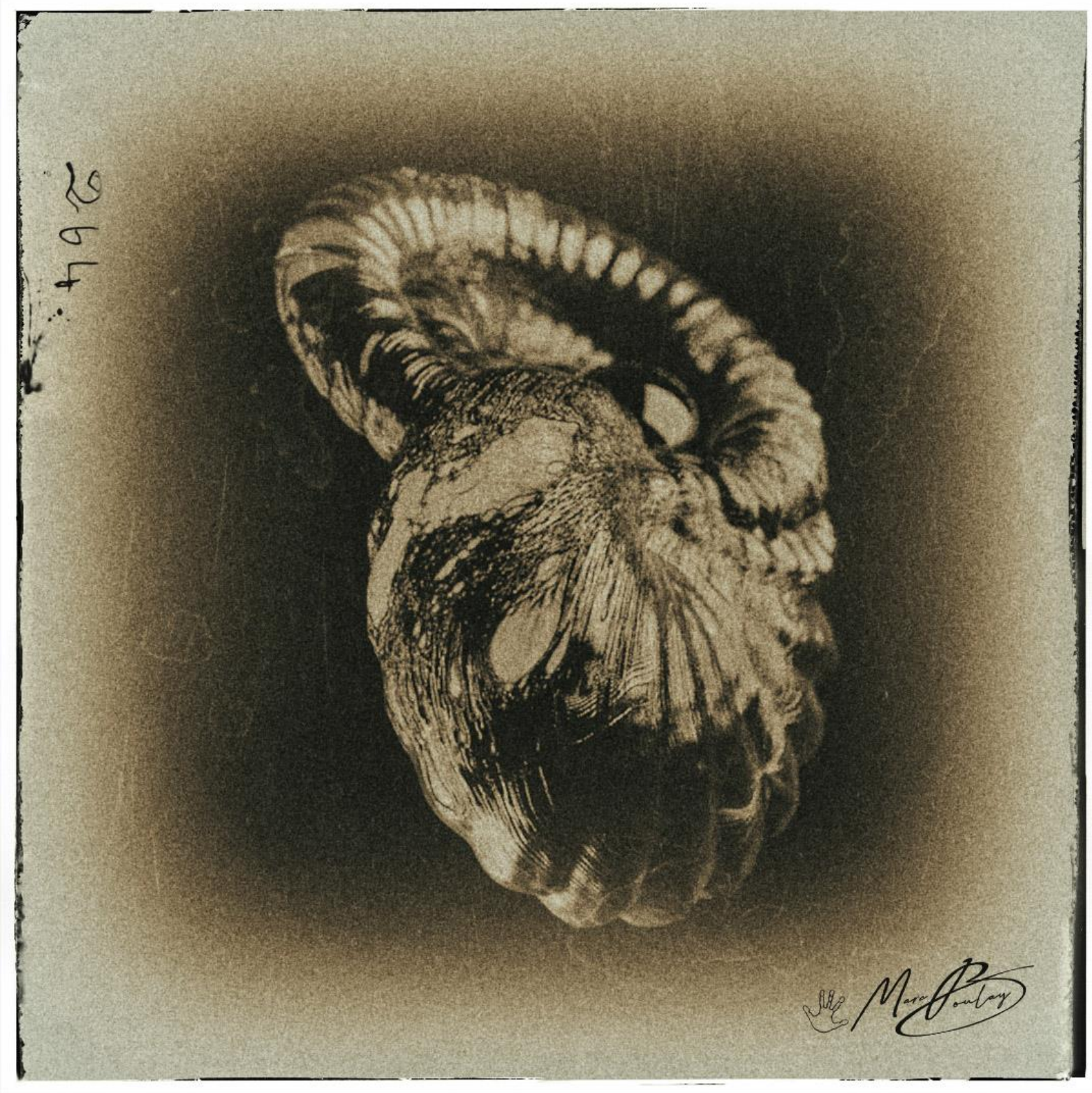




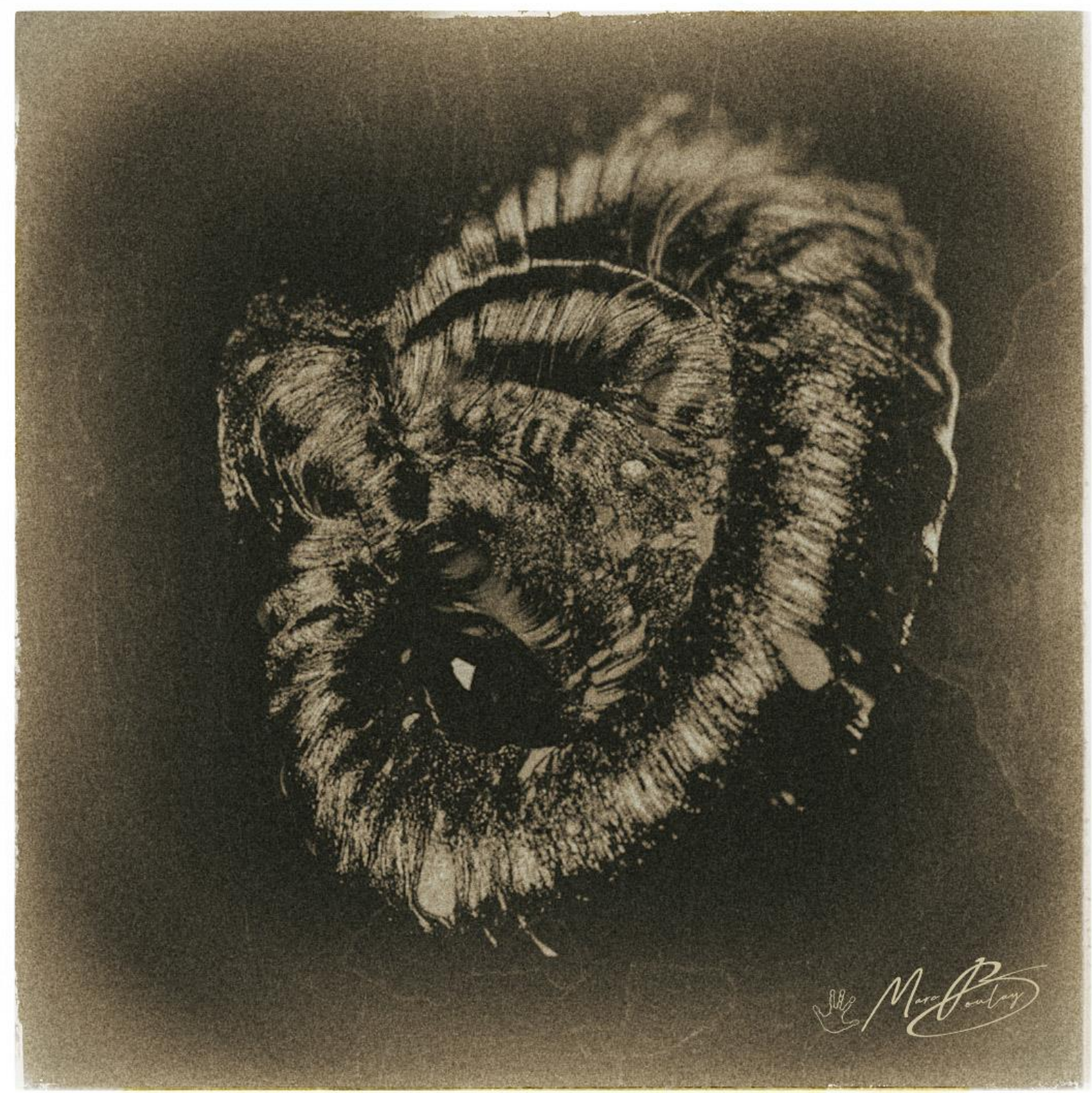




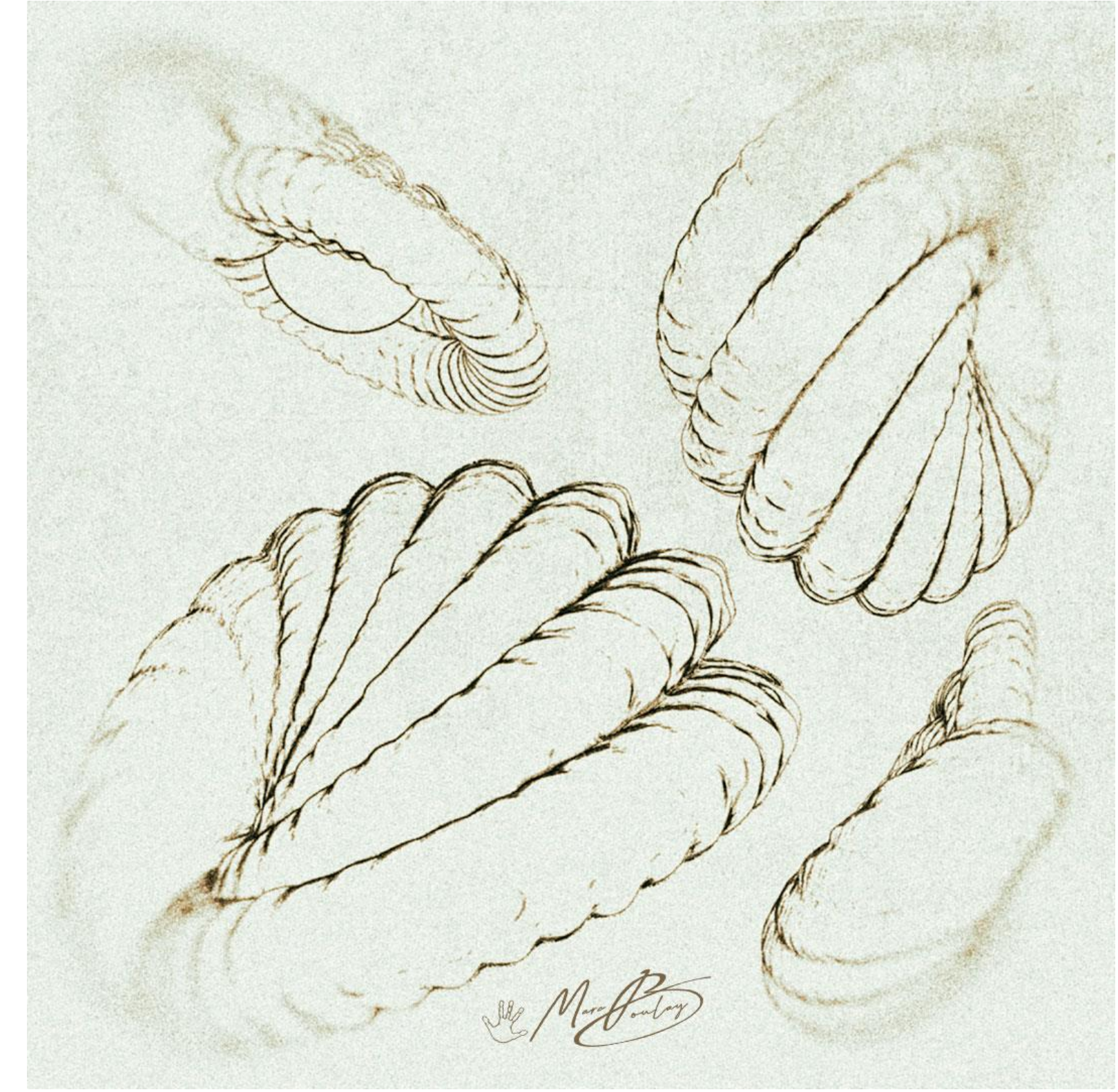

